\title{
Filterbank Reconstruction of Bandlimited Signals from Nonuniform and Generalized Samples
}

\author{
Yonina C. Eldar, Student Member, IEEE, and Alan V. Oppenheim, Fellow, IEEE
}

\begin{abstract}
This paper introduces a filterbank interpretation of various sampling strategies, which leads to efficient interpolation and reconstruction methods. An identity, which is referred to as the Interpolation Identity, is developed and is used to obtain particularly efficient discrete-time systems for interpolation of generalized samples as well as a class of nonuniform samples, to uniform Nyquist samples, either for further processing in that form or for conversion to continuous time. The Interpolation Identity also leads to new sampling strategies including an extension of Papoulis' generalized sampling expansion.
\end{abstract}

Index Terms-Filterbanks, generalized sampling, interpolation, nonuniform sampling, sampling.

\section{INTRODUCTION}

D ISCRETE-TIME signal processing (DSP) inherently relies on sampling a continuous time signal to obtain a discrete-time representation of the signal. The most common form of sampling used in the context of DSP is uniform (periodic) sampling. However, there are a variety of applications in which data is sampled in other ways, such as nonuniformly in time or through multichannel data acquisition. Examples in which nonuniform sampling may arise include data loss due to channel erasures and additive noise. Multichannel data can arise in digital flight control, where the velocity as well as the position are recorded. There are also applications where we can benefit from deliberately introducing more elaborate sampling schemes. Potential applications include data compression, efficient quantization methods [10], and flexible A/D converters.

Several extensions of the uniform sampling theorem are well known [5]. Specifically, it is well established that a bandlimited signal is uniquely determined from its nonuniform samples, provided that the average sampling rate exceeds the Nyquist rate [1]. However, in contrast to uniform sampling, reconstruction of a continuous-time signal from its nonuniform samples using the direct interpolation procedure is computationally difficult. Several alternative reconstruction methods from nonuniform samples have been previously suggested. These methods involve iterative algorithms (e.g., [2], [6], [14]), which are computationally demanding and have potential issues of convergence.

Manuscript received July 12, 1999; revised June 1, 2000. This work was supported in part through collaborative participation in the Advanced Sensors Consortium sponsored by the U.S. Army Research Laboratory under Cooperative Agreement DAAL01-96-2-0001 and supported in part by the Texas Instruments Leadership University Program. Y. Eldar is supported by an IBM Research Fellowship. The associate editor coordinating the review of this paper and approving it for publication was Dr. Xiang-Gen Xia.

The authors are with the Research Laboratory of Electronics, Massachusetts Institute of Technology, Cambridge, MA 02139 USA (e-mail: yonina@ mit.edu; avo@mit.edu).

Publisher Item Identifier S 1053-587X(00)07683-2.
Another well-known sampling theorem by Papoulis [8], which generalizes uniform sampling of a signal, states that a bandlimited signal can be reconstructed from uniformly spaced samples of the outputs of $M$ linear time-invariant (LTI) systems with the signal as their input sampled at one- $M$ th of the Nyquist rate. However, the reconstruction from these generalized samples is again computationally complex. In order to exploit alternative sampling methods in various applications, practical, efficient reconstruction algorithms are required.

Recently, there has been some work on sampling theorems for nonbandlimited signals [3], [13] and on nonuniform and generalized sampling theorems for discrete-time signals $[12$, Sec. 10.2].

Many of the algorithms for processing and analyzing a discrete-time signal assume that the signal corresponds to uniformly spaced samples of a continuous-time signal. When other sampling procedures are employed, a common approach is to interpolate to uniform Nyquist samples of the continuous-time signal prior to processing. Existing interpolation methods include approximate polynomial interpolation and iterative procedures [9]. Here again, practical, efficient interpolation algorithms are desirable.

In this paper, we derive an identity that leads to efficient reconstruction methods from generalized samples, as well as efficient interpolation to uniformly spaced samples. We then develop a new noniterative approach to reconstruction from recurrent and $N$ th-order nonuniform samples. The resulting procedure consists of processing the samples with a bank of LTI filters, either to reconstruct the original bandlimited continuous-time signal or to interpolate the nonuniform samples to uniformly spaced samples. In addition to offering efficient implementations, the filterbank framework leads to a new class of sampling strategies. As an example, we show that applying the identity derived in this paper to perfect reconstruction filterbanks results in a generalization of Papoulis' sampling theorem [8].

The organization of this paper is as follows. In Section II, we formulate the Interpolation Identity. Section III illustrates an application of the identity to sampling of a signal and its derivative. In Section IV, we describe recurrent nonuniform sampling and arrive at a continuous-time filterbank implementation of the reconstruction. We then apply the Interpolation Identity to convert the continuous-time filterbank to an equivalent discrete-time filterbank. The resulting discrete-time filterbank inherently interpolates the uniform samples of the continuous-time signal. In Section $\mathrm{V}$, we introduce a type of nonuniform sampling referred to as $N$ th-order nonuniform sampling. We then develop an efficient interpolation and reconstruction method from these samples using a rational discrete-time filterbank. Section VI demonstrates 


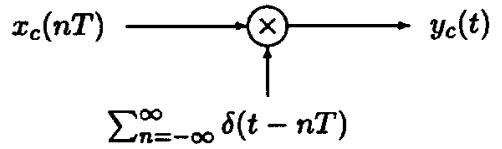

Fig. 1. Converting the sequence of samples $x_{c}(n T)$ to a continuous-time impulse train $y_{c}(t)$.

how the filterbank framework leads to new sampling strategies. In particular, we present a generalization to Papoulis' sampling theorem. In the various sections, key results are stated and their detailed derivation is included in the appropriate appendix.

\section{INTERPOLATION IDENTITY}

Throughout this paper, we use the variables $\Omega$ and $\omega$ to denote frequency variables for continuous-time and discrete-time, respectively. Capital letters are used to denote the Fourier transform, e.g., $X(\Omega)$ and $X(\omega)$ denote the continuous-time and discrete-time Fourier transforms of $x(t)$ and $x[n]$, respectively. Parentheses are used for continuous-time signals and brackets for discrete-time signals. To further distinguish between continuous-time and discrete-time signals, we will usually denote the former with a subscript, e.g., $x_{c}(t)$. We assume that all signals have finite energy and are bandlimited to $W$, i.e., their Fourier transform is zero for $W \leq|\Omega| . T_{Q}$ denotes the Nyquist period given by $T_{Q}=\pi / W$. We use the notation in the block diagram of Fig. 1 to denote conversion of the sequence of samples $x_{c}(n T)$ to a continuous-time impulse train $y_{c}(t)$, where $y_{c}(t)=\sum_{n=-\infty}^{\infty} x_{c}(n T) \delta(t-n T)$. We refer to this operation as impulse modulation.

The following equivalence, which we refer to as the Interpolation Identity, will be used in subsequent sections to arrive at efficient implementations of the reconstruction from generalized and nonuniformly spaced samples. The proof of this identity is given in Appendix A.

Interpolation Identity: Let $x_{c}(t)$ be a finite energy continuous-time signal bandlimited to $W=\pi / T_{Q}$, and let $h_{l}(t), l=0,1, \cdots, M-1$ denote the impulse responses of the continuous-time filters with corresponding frequency responses $H_{l}(\Omega), l=0,1, \cdots, M-1$ bandlimited to $W$. For any $M$ and $T$ such that $T / T_{Q}-1 / M=k$ for some integer $k$, the block diagrams depicted in Fig. 2(a) and (b) are equivalent for

$$
\begin{aligned}
\tilde{H}(\omega)= & \frac{M}{T_{Q}} \sum_{l=0}^{M-1}\left(H_{l}\left(\frac{M \omega}{T_{Q}}-\frac{2 \pi l}{T_{Q}}\right)\right. \\
& \left.+H_{l}\left(\frac{M \omega}{T_{Q}}-\frac{2 \pi(l-M)}{T_{Q}}\right)\right), \quad|\omega| \leq \pi
\end{aligned}
$$

The block diagram of Fig. 2(b) consists of expanding a sequence of samples by a factor of $M T / T_{Q}$ and then filtering by a discrete-time filter with frequency response given by (1). The filtered output is then decimated by a factor of $M$ followed by impulse modulation and lowpass filtering. The input-output relation for the expander is given by

$$
x_{e}[n]= \begin{cases}x\left[\frac{n T_{Q}}{M T}\right], & n=0, \pm M T / T_{Q}, \pm 2 M T / T_{Q}, \cdots \\ 0, & \text { otherwise }\end{cases}
$$

The input-output relation for the decimator is given by

$$
y_{d}[n]=y_{e}[n M]
$$

For the case $M=1$ and $T=N T_{Q}$, the Interpolation Identity reduces to the equivalence depicted in Fig. 3(a) and (b), where

$$
\tilde{H}(\omega)=\frac{1}{T_{Q}} H\left(\frac{\omega}{T_{Q}}\right), \quad|\omega| \leq \pi
$$

Note that (4) implies that $\tilde{h}[n]=h\left(n T_{Q}\right)$, where $\tilde{h}[n]$ is the discrete-time impulse response with frequency response $\tilde{H}(\omega)$, and $h(t)$ is the continuous-time impulse response with frequency response $H(\Omega)$. Since $T=N T_{Q}$, the sequence $x_{c}(n T)$ is, in general, an undersampled representation of $x_{c}(t)$, and consequently, $y_{c}(t)$ is, in general, a filtered and aliased version of $x_{c}(t)$.

As an illustration of the use of the identity, we apply it in the next section to a well-known sampling theorem associated with the reconstruction from uniform samples of a signal and its derivative. Through the use of the identity, we obtain a particularly efficient system for interpolation of the samples to uniform Nyquist samples, either for further processing in that form or for conversion to continuous time. In Sections IV and V, we apply the identity to two classes of nonuniform sampling strategies referred to as recurrent and $N$ th-order nonuniform sampling. In Section VI, the Interpolation Identity is used to generate new classes of sampling theorems.

\section{INTERPOLATION AND RECONSTRUCTION FROM SAMPLES OF A SIGNAL AND ITS DERIVATIVE}

As an example of the application of the Interpolation Identity, consider sampling a signal and its derivative. It is well known that a bandlimited signal can be reconstructed from uniform samples of the signal and its derivative at half the Nyquist rate [4] using the reconstruction formula

$$
\begin{aligned}
x_{c}(t)= & \sum_{k=-\infty}^{\infty} \operatorname{sinc}^{2}\left(\pi\left(t-k 2 T_{Q}\right) / 2 T_{Q}\right) \\
& \cdot\left(f[k]+\left(t-k 2 T_{Q}\right) f^{\prime}[k]\right)
\end{aligned}
$$

where $f[n]=x_{c}\left(n 2 T_{Q}\right)$, and $f^{\prime}[n]=d x_{c}(t) /\left.d t\right|_{t=n 2 T_{Q}}$. Note that the sequences $f[n]$ and $f^{\prime}[n]$ are undersampled representations of $x_{c}(t)$ and $d x_{c}(t) / d t$, respectively.

Equation (5) can be implemented using the continuous-time filterbank depicted in Fig. 4, with $h_{0}(t)=\operatorname{sinc}^{2}\left(\pi t / 2 T_{Q}\right)$ and $h_{1}(t)=t \operatorname{sinc}^{2}\left(\pi t / 2 T_{Q}\right)$. Note that both filters in Fig. 4 are bandlimited to $W=\pi / T_{Q}$. If, instead of reconstructing $x_{c}(t)$, we are interested in interpolating the uniform Nyquist samples of $x_{c}(t)$ from $f[n]$ and $f^{\prime}[n]$, the interpolation formula obtained from substituting $t=n T_{Q}$ in (5) is

$$
\begin{aligned}
x[n] & =x_{c}\left(n T_{Q}\right) \\
& =\sum_{k=-\infty}^{\infty} \operatorname{sinc}^{2}(\pi(n-2 k) / 2)\left(f[k]+T_{Q}(n-2 k) f^{\prime}[k]\right) .
\end{aligned}
$$




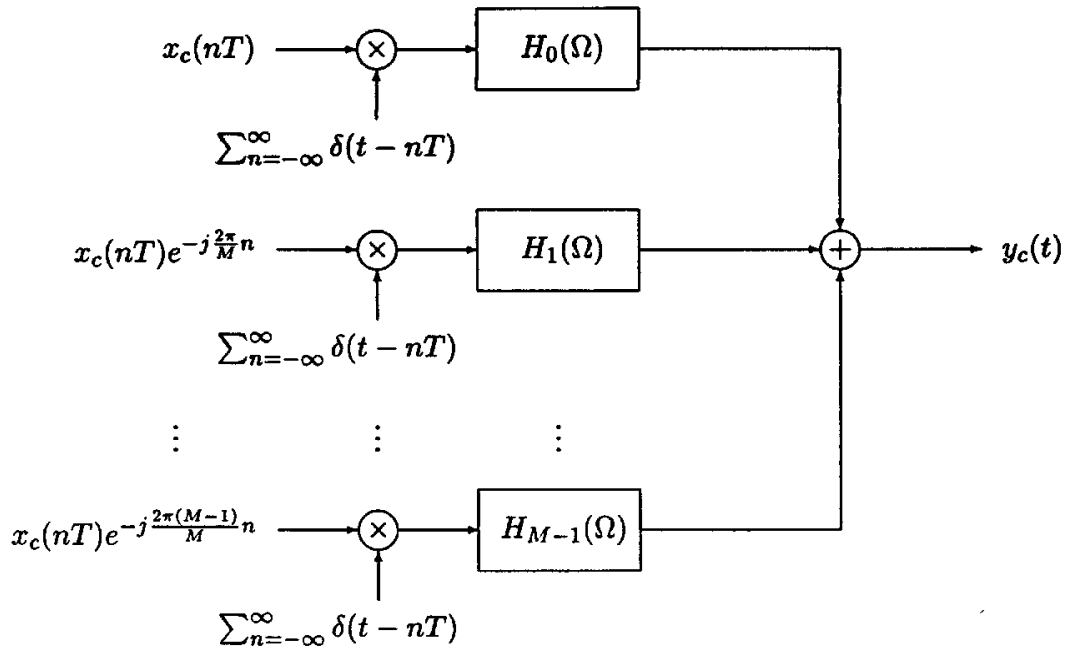

(a)

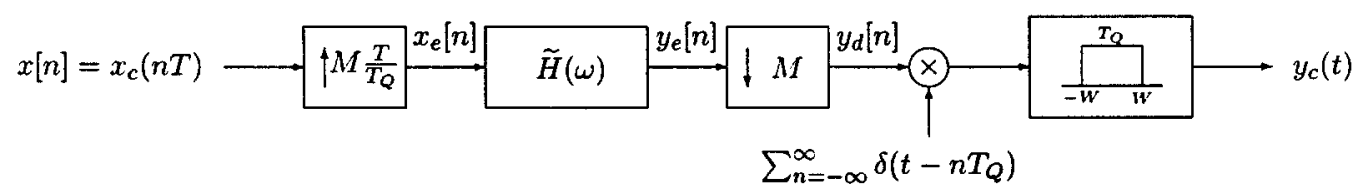

(b)

Fig. 2. Interpolation Identity.

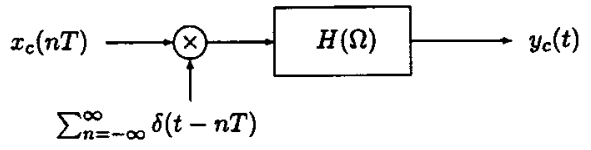

(a)

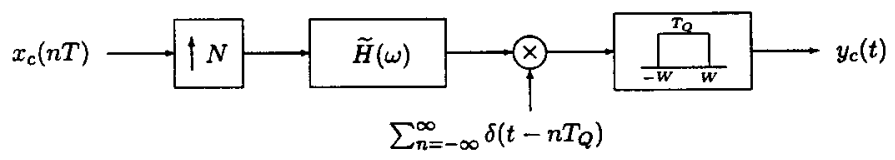

(b)

Fig. 3. Interpolation Identity for the case $M=1$ and $T=N T_{Q}$.

Reconstruction of $x_{c}(t)$ using (5) or interpolation using (6) are both difficult to implement directly. However, both interpolation and reconstruction can be implemented in a simpler form by applying the Interpolation Identity to the system in Fig. 4. Specifically, the continuous-time filterbank of Fig. 4 can be converted to a discrete-time filterbank followed by a continuous-time lowpass filter (LPF). Applying the equivalence of Fig. 3 to each branch in Fig. 4 and moving the identical impulse train modulation and LPF in each branch outside the summer, we obtain the equivalent implementation in Fig. 5, where $\tilde{h}_{p}[n]=h_{p}\left(n T_{Q}\right)$ for $p=0,1$. As with the continuous-time filterbank, the overall output of Fig. 5 is the original continuous-time signal $x_{c}(t)$. Since $x_{c}(t)$ is reconstructed through lowpass filtering of a uniformly spaced impulse train with period $T_{Q}$, the impulse train values $x[n]$ must correspond to uniformly spaced samples of $x_{c}(t)$ at the Nyquist rate. Thus, we conclude that the discrete-time filterbank provides a dis-

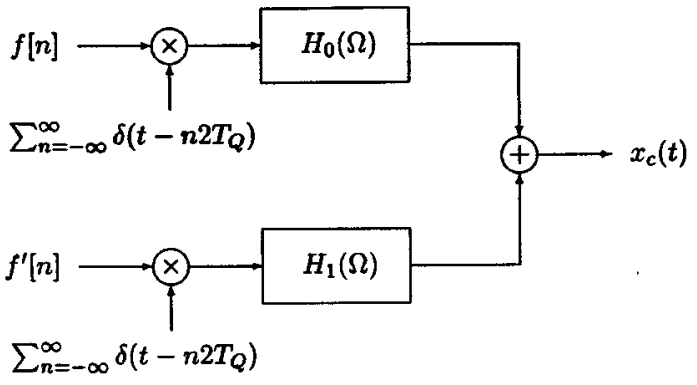

Fig. 4. Reconstruction from samples of a signal and its derivative at half the Nyquist rate.

crete-time mechanism for converting the uniform generalized samples of the signal and its derivative to uniform Nyquist samples. The filterbank can be implemented very efficiently, exploiting the many known results regarding efficient implementation of the filters comprising a discrete-time filterbank (see, e.g., [12]).

By following an analogous procedure, we can arrive at efficient interpolation and reconstruction methods for other forms of generalized samples. In the next section, we focus on efficient implementation of the reconstruction from recurrent nonuniform samples using a bank of continuous-time and discrete-time filters.

\section{RECURRENT NONUNIFORM SAMPLING}

It is well established that a continuous-time signal $x_{c}(t)$ can be reconstructed from its samples at a set of sampling times $\left\{t_{n}\right\}$ if the average sampling period is smaller than the 


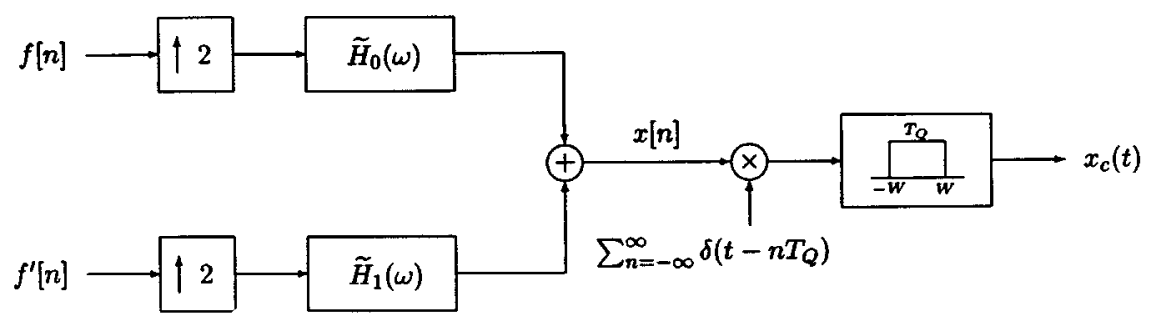

Fig. 5. Interpolation and reconstruction using a discrete-time filterbank.

Nyquist period, where the average sampling period is defined as $\lim _{n \rightarrow \infty}\left(t_{n} / n\right)$. The essential result is incorporated in the following theorem by Yao and Thomas [15].

Theorem 1: Let $x_{c}(t)$ be a finite energy bandlimited signal such that $X_{c}(\Omega)=0$ for $|\Omega|>W-\epsilon$ for some $0<\epsilon \leq W$. $x_{c}(t)$ is uniquely determined by its samples $x_{c}\left(t_{n}\right)$ if

$$
\begin{aligned}
& \left|t_{n}-n \frac{\pi}{W}\right|<L<\infty \\
& \left|t_{n}-t_{m}\right|>\delta>0, \quad n \neq m .
\end{aligned}
$$

The reconstruction is given by

$$
x_{c}(t)=\sum_{n=-\infty}^{\infty} x_{c}\left(t_{n}\right) \frac{G(t)}{G^{\prime}\left(t_{n}\right)\left(t-t_{n}\right)}
$$

where

$$
G(t)=\left(t-t_{0}\right) \prod_{\substack{n=-\infty \\ n \neq 0}}^{\infty}\left(1-\frac{t}{t_{n}}\right)
$$

$G^{\prime}\left(t_{n}\right)$ is the derivative of $G(t)$ evaluated at $t=t_{n}$, and if $t_{n}=0$ for some $n$, then $t_{0}=0$.

Reconstruction from nonuniform samples using (8) directly is considerably more complex than reconstruction from uniform samples. In this section, we focus on an efficient implementation of (8) for the case of recurrent nonuniform sampling. In this form of sampling, the sampling points are divided into groups of $N$ points each. The groups have a recurrent period, which is denoted by $T$, that is equal to $N$ times the Nyquist period $T_{Q}$. Each period consists of $N$ nonuniform sampling points. Denoting the points in one period by $t_{p}, p=0,1, \cdots, N-1$, the complete set of sampling points are

$$
t_{p}+n T, \quad p=0,1, \cdots, N-1, n \in(-\infty, \infty)
$$

where $T=N T_{Q}$. Without loss of generality, we will assume throughout that $t_{0}=0$.

Recurrent nonuniform samples can be regarded as a combination of $N$ sequences of uniform samples taken at one $N$ th of the Nyquist rate. An example of a sampling distribution for the case $N=3$ is depicted in Fig. 6 .

Recurrent nonuniform sampling arises in a broad range of applications. For example, we might consider converting a continuous-time signal to a discrete-time signal using a series of A/D converters, each operating at a rate lower than the Nyquist rate, such that the average sampling rate is equal to the Nyquist

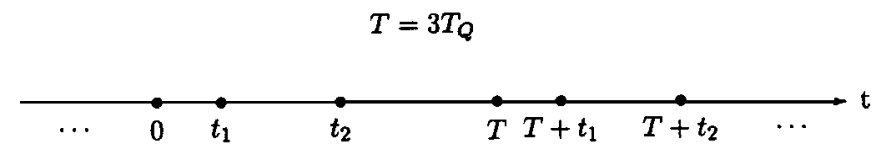

Fig. 6. Sampling distribution for $N=3$.

rate. This may be beneficial in applications where high-rate A/D converters are required. Typically, the cost and complexity of a converter will increase (more than linearly) with the rate. In such cases, we can benefit from converting a continuous-time signal to a discrete-time signal using $N \mathrm{~A} / \mathrm{D}$ converters, each operating at one $N$ th of the Nyquist rate. Since the converters are typically not synchronized, the resulting discrete-time signal is a combination of $N$ sequences of uniform samples, where each sequence corresponds to samples at one $N$ th of the Nyquist rate of a time delayed version of the continuous-time signal. Thus, the resulting discrete-time signal corresponds to recurrent nonuniform samples of the continuous-time signal.

Dividing the time axis into nonoverlapping intervals of length $N T_{Q}$, every interval contains $N$ sampling points, which implies that the average sampling rate is the Nyquist rate. Based on Theorem 1, we can therefore reconstruct a continuous-time signal $x_{c}(t)$ from its recurrent nonuniform samples $x_{c}\left(t_{n}\right)$, where the sampling times $\left\{t_{n}\right\}$ are given by (10). In particular, substituting (10) in (8) and (9), we obtain the following reconstruction formula (see Appendix B):

$$
\begin{aligned}
x_{c}(t)= & \sum_{n=-\infty}^{\infty} \sum_{p=0}^{N-1} x_{c}\left(n T+t_{p}\right) \\
& \cdot \frac{a_{p}(-1)^{n N} \prod_{q=0}^{N-1} \sin \left(\pi\left(t-t_{q}\right) / T\right)}{\pi\left(t-n T-t_{p}\right) / T}
\end{aligned}
$$

where

$$
a_{p}=\frac{1}{\prod_{q=0, q \neq p}^{N-1} \sin \left(\pi\left(t_{p}-t_{q}\right) / T\right)} .
$$

As with (8), direct implementation of (11) is computationally difficult. We will now develop new, efficient, noniterative implementations of (11). In Section IV-A, we develop an implementation that consists of processing the samples with a bank of continuous-time LTI filters. In Section IV-B, we develop an alternative implementation using a bank of discrete-time LTI filters. 


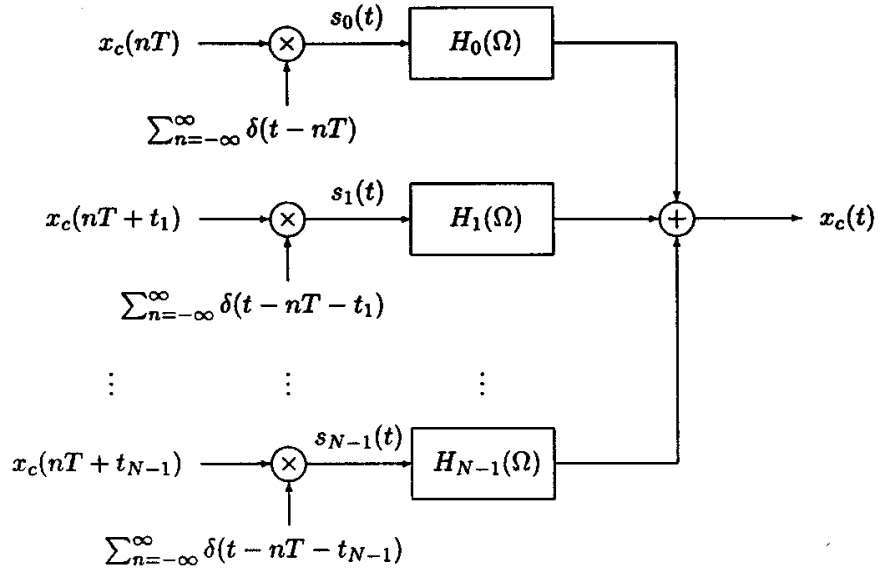

Fig. 7. Reconstruction from recurrent nonuniform samples using a continuous-time filterbank.

\section{A. Reconstruction from Recurrent Nonuniform Samples Using a Continuous-Time Filterbank}

In this subsection, we develop a continuous-time filterbank representation of (11). To this end, we interchange the order of summations in (11) and denote the inner sum by $f_{p}(t)$, i.e.,

$$
f_{p}(t)=\sum_{k=-\infty}^{\infty} x_{c}\left(k T+t_{p}\right) \frac{a_{p}(-1)^{k N} \prod_{q=0}^{N-1} \sin \left(\pi\left(t-t_{q}\right) / T\right)}{\pi\left(t-k T-t_{p}\right) / T} .
$$

Using the relation $\sin (t-k \pi)=(-1)^{k} \sin (t)$, we can express $f_{p}(t)$ as a convolution. Specifically

$$
f_{p}(t)=s_{p}(t) * h_{p}(t)
$$

where

$$
h_{p}(t)=a_{p} \frac{\prod_{q=0}^{N-1} \sin \left(\pi\left(t+t_{p}-t_{q}\right) / T\right)}{\pi t / T}
$$

and $s_{p}(t)$ is an impulse train of samples, i.e.,

$$
s_{p}(t)=\sum_{k=-\infty}^{\infty} x_{c}\left(k T+t_{p}\right) \delta\left(t-k T-t_{p}\right)
$$

$x_{c}(t)$ given by (11) can now be expressed as a sum of $N$ convolutions:

$$
x_{c}(t)=\sum_{p=0}^{N-1} s_{p}(t) * h_{p}(t) .
$$

Equation (17) can be interpreted as a continuous-time filterbank as depicted in Fig. 7. The signals $s_{p}(t)$ are formed according to (16), i.e., the samples are divided into $N$ subsequences, where each subsequence corresponds to samples at one- $N$ th of the Nyquist rate of a time-shifted version of $x_{c}(t)$. Each subsequence is converted to a continuous-time signal $s_{p}(t)$ using a shifted impulse train. The signal $s_{p}(t)$ is then filtered

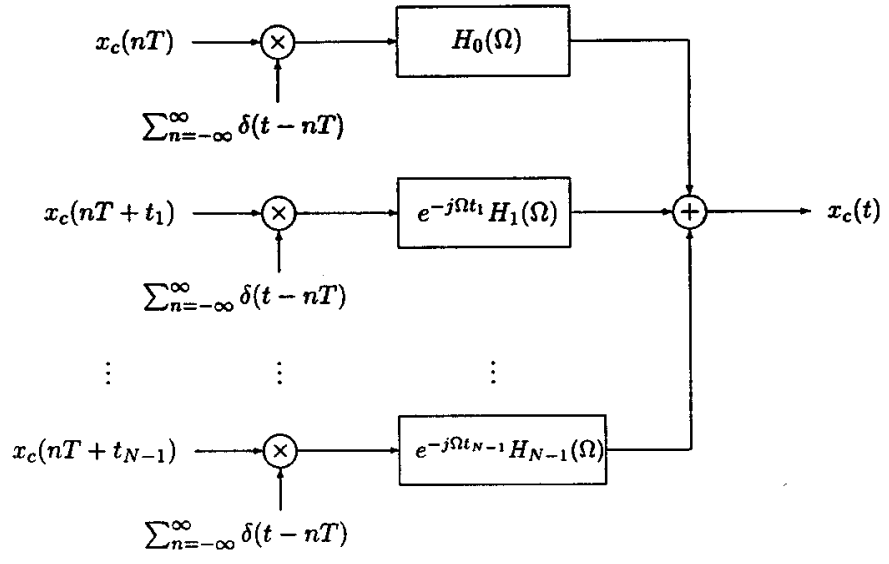

Fig. 8. Alternative form of Fig. 7.

by a continuous-time filter with impulse response $h_{p}(t)$ given by (15). Summing the outputs of the $N$ branches results in the reconstructed signal $x_{c}(t)$.

Note that each one of the subsequences corresponds to uniform samples at one-Nth of the Nyquist rate. Therefore, the output of each branch of the filter bank is an aliased and filtered version of $x_{c}(t)$. The filters, as specified by (15), have the inherent property that the aliasing components of the filter outputs cancel in forming the summed output $x_{c}(t)$.

An alternative form of Fig. 7, which we will find useful in Section IV-B, is shown in Fig. 8. This form follows in a straightforward way by simply noting that the delay of $t_{p}$ in the impulse train of the $p$ th branch can be incorporated into the filter $h_{p}(t)$.

To determine the frequency responses of the filters in Figs. 7 and 8 , we note that the impulse response given by (15) can be expressed as

$$
\begin{aligned}
h_{p}(t) & =a_{p} T \frac{\sin (\pi t / T)}{\pi t} \prod_{\substack{q=0 \\
q \neq p}}^{N-1} \sin \left(\pi\left(t+t_{p}-t_{q}\right) / T\right) \\
& =a_{p} T \frac{\sin (\pi t / T)}{\pi t} \sum_{k=-N+1}^{N-1} b_{k} e^{j k W t / N}
\end{aligned}
$$

where the complex coefficients $b_{k}$ are the result of expanding the product of sines in (18) into complex exponentials.

The first term in (19) $\sin (\pi t / T) / \pi t$ corresponds to an ideal LPF with cut-off frequency $W / N$, which we denote as $H_{\mathrm{LPF}}(\Omega ; W / N)$. The effect of the summation is to create shifted and scaled versions of the LPF, i.e.,

$$
H_{p}(\Omega)=a_{p} T \sum_{k=-N+1}^{N-1} b_{k} H_{\mathrm{LPF}}\left(\Omega-\frac{k W}{N} ; \frac{W}{N}\right) .
$$

Hence, we conclude that the filters $H_{p}(\Omega)$ in Figs. 7 and 8 have the properties that $H_{p}(\Omega)=0$ for $|\Omega|>W$, i.e., the filters are bandlimited to the same bandwidth as the continuous-time signal, and each filter $H_{p}(\Omega)$ is piecewise constant over frequency intervals of length $2 W / N$.

In the next subsection, we will derive a discrete-time filter bank implementation of the reconstruction, which also provides efficient interpolation to uniform samples. 


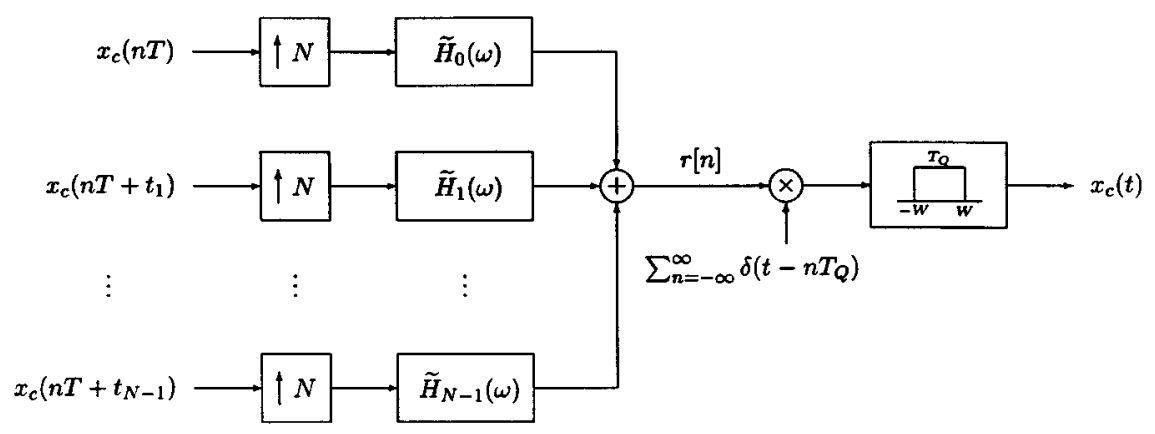

Fig. 9. Reconstruction from recurrent nonuniform samples using a discrete-time filterbank.

\section{B. Interpolation and Reconstruction from Recurrent Nonuniform Samples Using a Discrete-Time Filterbank}

Following an analogous procedure to Section III, the continuous-time filterbank of Fig. 8 can be converted to a discrete-time filterbank followed by a continuous-time LPF. Applying the Interpolation Identity of Fig. 3 to each branch in Fig. 8 and moving the impulse modulation and LPF in each branch outside the summer, we obtain the equivalent implementation in Fig. 9, where

$$
\tilde{H}_{p}(\omega)=\frac{1}{T_{Q}} H_{p}\left(\frac{\omega}{T_{Q}}\right) e^{-j t_{p} \omega / T_{Q}}, \quad|\omega| \leq \pi
$$

for $p=0,1, \cdots, N-1$.

As with the continuous-time filter banks of Figs. 7 and 8, the overall output of Fig. 9 is the original continuous-time signal $x_{c}(t)$. Furthermore, since $x_{c}(t)$ is reconstructed through lowpass filtering of a uniformly spaced impulse train with period $T_{Q}$, the impulse train values $r[n]$ must correspond to uniformly spaced samples of $x_{c}(t)$ at the Nyquist rate. Thus, the discrete-time filterbank of Fig. 9 effectively interpolates the recurrent nonuniform samples to uniform Nyquist samples.

The discrete-time filterbank of Fig. 9 can be used to interpolate the uniform samples and to reconstruct the continuous-time signal from its recurrent nonuniform samples very efficiently, exploiting the many known results regarding the implementation of filterbank structures. As with the continuous-time filterbank, the magnitude responses of the discrete-time filters are piecewise constant, which allows for further efficiency in the implementation.

\section{NTH-ORDER NONUNIFORM SAMPLING}

In this section, we consider a subclass of recurrent nonuniform sampling, for which the sampling points in each period can be further divided into groups with a common inner period. Specifically, the samples consist of $N$ sequences of uniform samples taken at $N$ rates, i.e., the set of sampling points are

$$
t_{p}+n T_{p}, \quad p=0,1, \cdots, N-1, n \in(-\infty, \infty)
$$

where $\sum_{p=0}^{N-1} 1 / T_{p}=1 / T_{Q}$ so that the average sampling rate is the Nyquist rate. An example of a sampling distribution for the case $N=2$ with $T_{0}=3 T_{Q}$ and $T_{1}=3 T_{Q} / 2$ is depicted in Fig. 10.

$N$ th-order nonuniform sampling may arise in a similar context as recurrent nonuniform sampling, namely, when

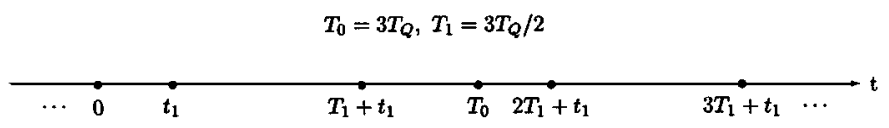

Fig. 10. Sampling distribution for $N=2$ with $T_{0}=3 T_{Q}$ and $T_{1}=3 T_{Q} / 2$.

converting a continuous-time signal to a discrete-time signal using a series of $\mathrm{A} / \mathrm{D}$ converters, each operating at a different rate, such that the average sampling rate is equal to the Nyquist rate. Thus, in addition to allowing for a series of asynchronized A/D converters as in recurrent nonuniform sampling, we also allow for converters operating at different rates.

The reconstruction formula is given by (23), shown at the bottom of the next page (see Appendix C). If $T_{p} \neq T_{q}$ for some $p$ and $q$, (23) cannot be expressed as a sum of $N$ convolutions. Nevertheless, we will show that (23) can be implemented using $N$ discrete-time filters and a continuous-time LPF.

To this end, we denote the inner sum in (23) by $g_{p}(t)$, i.e., in (24), shown at the bottom of the next page. We further denote by $T$ the least common multiplier (LCM) of $\left\{T_{p}\right\}$ and define $M_{p}=$ $T / T_{p}$. In Appendix D, we show that $g_{p}(t)$ can be expressed as a sum of $M_{p}$ convolutions, i.e.,

$$
g_{p}(t)=\sum_{m=0}^{M_{p}-1} s_{p m}(t) * h_{p m}(t) .
$$

Here

$$
h_{p m}(t)=\sum_{l=0}^{M_{p}-1} \frac{1}{M_{p}} e^{j\left(2 \pi l / M_{p}\right) m} f_{p l}\left(t-t_{p}\right)
$$

where we define $f_{p k}(t)$ as

$$
\begin{aligned}
& f_{p k}(t)= \\
& \frac{T_{p} \sin \left(\pi t / T_{p}\right) \prod_{q=0, q \neq p}^{N-1} \sin \left(\pi\left(t+t_{p}-t_{q}\right) / T_{q}+\pi k T_{p} / T_{q}\right)}{\pi t \prod_{q=0, q \neq p}^{N-1} \sin \left(\pi\left(t_{p}-t_{q}\right) / T_{q}+\pi k T_{p} / T_{q}\right)}
\end{aligned}
$$

and $s_{p m}(t)$ is an impulse train of modulated samples, i.e.,

$$
s_{p m}(t)=\sum_{k=-\infty}^{\infty} x_{c}\left(k T_{p}+t_{p}\right) e^{-j\left(2 \pi m / M_{p}\right) k} \delta\left(t-k T_{p}\right)
$$


Equation (25) can be interpreted as a filterbank, as depicted in Fig. 11. The signals $s_{p m}(t)$ are formed according to (28), i.e., the samples are divided into $N$ subsequences, where each subsequence corresponds to uniform samples with period $T_{p}$ of a time-shifted version of the original signal. From each subsequence $x_{c}\left(n T_{p}+t_{p}\right)$, we form $M_{p}$ discrete-time signals by modulating the subsequence by $e^{-j\left(2 \pi m / M_{p}\right) n}$ for $m=0,1, \cdots, M_{p}-1$. For every $p$, each one of the $M_{p}$ discrete-time signals is converted to a continuous-time signal $s_{p m}(t)$ using an impulse train with period $T_{p}$. The signal $s_{p m}(t)$ is then filtered by a continuous-time filter with impulse response given by (26) and (27). Summing the outputs of the $M_{p}$ branches results in $g_{p}(t)$. Since $x_{c}(t)=\sum_{p=0}^{N-1} g_{p}(t)$, summing the outputs of $N$ filterbanks of the form of Fig. 11 will result in $x_{c}(t)$. Thus, (23) can be implemented using $\sum_{p=0}^{N-1} M_{p}$ continuous-time filters (where, in general, $\sum_{p=0}^{N-1} M_{p}>N$ ).

The filters in Fig. 11, with impulse responses $h_{p m}(t)$ given by (26), are all bandlimited to $W$ since $f_{p k}(t)$ given by (27) is bandlimited to $W$ for all $p$ and $k$. This can be seen by rewriting $f_{p k}(t)$ as

$$
\begin{aligned}
f_{p k}(t)= & a_{p k} \frac{\sin \left(\pi t / T_{p}\right)}{\pi t} \prod_{q=0, q \neq p}^{N-1} \\
& \cdot \sin \left(\pi\left(t+t_{p}-t_{q}\right) / T_{q}+\pi k T_{p} / T_{q}\right)
\end{aligned}
$$

where $a_{p k}$ is a constant. The term $p(t)=\sin \left(\pi t / T_{p}\right) / \pi t$ corresponds to an ideal LPF with cutoff frequency $\pi / T_{p}$. By expanding the product of sines into complex exponentials, we see that the effect of the product is to create shifted and scaled versions of the LPF. The largest shift will result when multiplying $p(t)$ by $b_{p k} \exp \left(-j \pi t \sum_{q, q \neq p} 1 / T_{q}\right)$, where $b_{p k}$ is a constant. This corresponds to shifting the LPF by $\omega_{0}=\pi \sum_{q, q \neq p} 1 / T_{q}$ in the frequency domain. Since the cutoff frequency of the LPF is $\pi / T_{p}, f_{p k}(t)$ is bandlimited to $\omega_{0}+\pi / T_{p}=\pi \sum_{q=0}^{N-1} 1 / T_{q}=$ $\pi / T_{Q}=W$. From (26), it then follows that the filters in Fig. 11 are all bandlimited to $W$ as well.

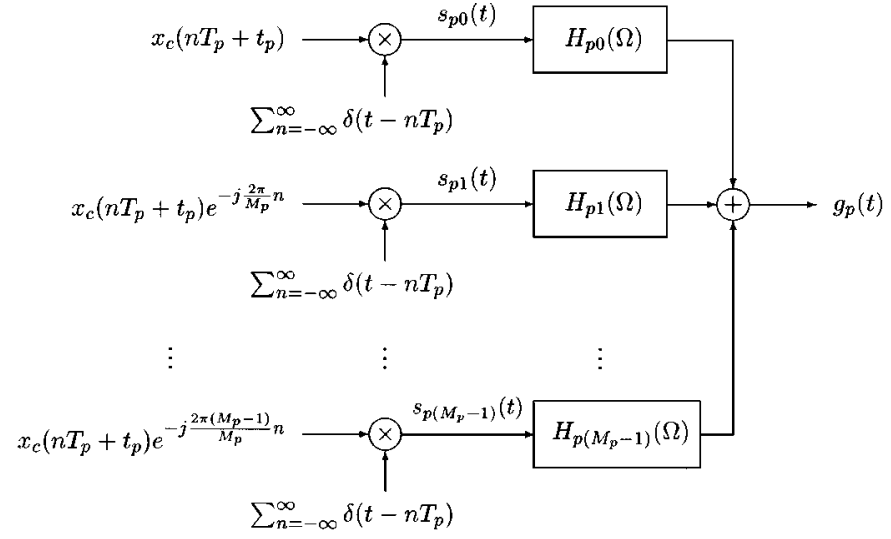

Fig. 11. Reconstructing $g_{p}(t)$ using a continuous-time filterbank.

We now wish to obtain an equivalent implementation of (23) using $N$ discrete-time filters. We first assume that each sampling period $T_{p}$ satisfies the condition

$$
T_{p} / T_{Q}-1 / M_{p}=k_{p}
$$

for some integer $k_{p}$. Then, since $x_{c}(t)$ is obtained by summing the outputs of $N$ filterbanks of the form of Fig. 11 and each sampling period $T_{p}$ satisfies (30), we can apply the Interpolation Identity to each filterbank, resulting in $N$ branches of the form of Fig. 2(b). Moving the impulse modulation and LPF in each branch outside the summer and noting that $M_{p} T_{p} / T_{Q}=T / T_{Q}$ for all $p$ leads to the equivalent implementation of Fig. 12, where

$$
\begin{aligned}
\tilde{H}_{p}(\omega)= & \frac{M_{p}}{T_{Q}} \sum_{l=0}^{M_{p}-1}\left(H_{p l}\left(\frac{M_{p} \omega}{T_{Q}}-\frac{2 \pi l}{T_{Q}}\right)\right. \\
& \left.+H_{p l}\left(\frac{M_{p} \omega}{T_{Q}}-\frac{2 \pi\left(l-M_{p}\right)}{T_{Q}}\right)\right), \quad|\omega| \leq \pi
\end{aligned}
$$

and $H_{p l}(\Omega)$ is the frequency response of the filter $h_{p l}(t)$ given by (26). The overall output of Fig. 12 is the original continuous-time

$$
x_{c}(t)=\sum_{p=0}^{N-1} \sum_{n=-\infty}^{\infty} x_{c}\left(n T_{p}+t_{p}\right) \frac{T_{p} \prod_{q=0}^{N-1} \sin \left(\pi\left(t-t_{q}\right) / T_{q}\right)}{\pi(-1)^{n} \prod_{q=0, q \neq p}^{N-1} \sin \left(\pi\left(t_{p}-t_{q}\right) / T_{q}+\pi n T_{p} / T_{q}\right)\left(t-n T_{p}-t_{p}\right)} .
$$

$$
g_{p}(t)=\sum_{k=-\infty}^{\infty} x_{c}\left(k T_{p}+t_{p}\right) \frac{T_{p} \prod_{q=0}^{N-1} \sin \left(\pi\left(t-t_{q}\right) / T_{q}\right)}{\pi(-1)^{k} \prod_{q=0, q \neq p}^{N-1} \sin \left(\pi\left(t_{p}-t_{q}\right) / T_{q}+\pi k T_{p} / T_{q}\right)\left(t-k T_{p}-t_{p}\right)} .
$$




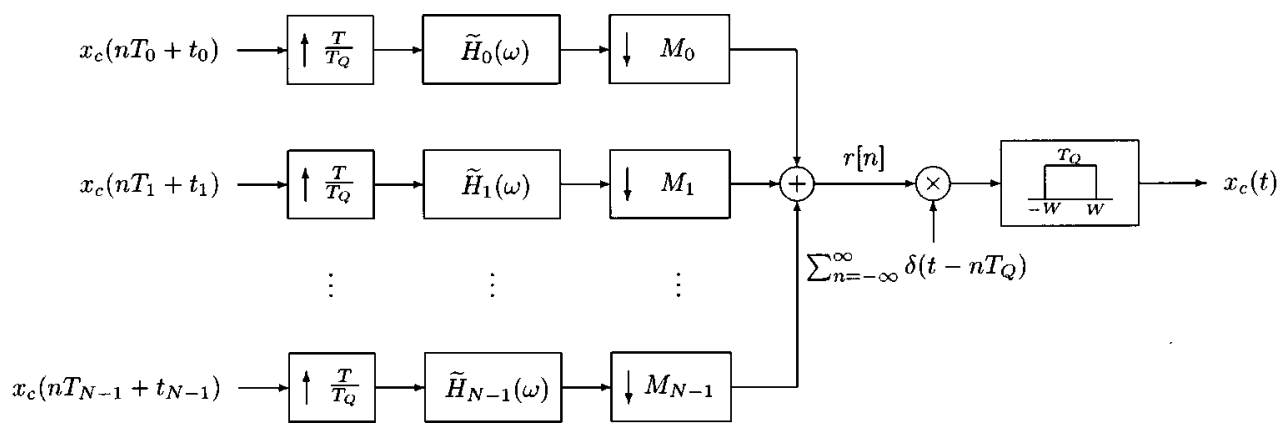

Fig. 12. Reconstruction from $N$ th-order nonuniform samples using a discrete-time filterbank.

signal $x_{c}(t)$, and the impulse train values $r[n]$ correspond to uniformly spaced samples of $x_{c}(t)$ at the Nyquist rate.

Using the discrete-time filterbank of Fig. 12, we can reconstruct the continuous-time signal from its $N$ th-order samples very efficiently using only $N$ discrete-time filters, in contrast with the continuous-time implementation that requires $\sum_{p=0}^{N-1} M_{p}$ filters. In addition, the discrete-time filterbank interpolates the uniform Nyquist samples from the $N$ th-order nonuniform samples.

We now show that we can implement (23) using $N$ discrete-time filters as in Fig. 12 for any set of sampling periods. This stems from the fact that if a continuous-time signal $x_{c}(t)$ is bandlimited to $W=\pi / T_{Q}$, then it is also bandlimited to $W^{\prime}=\pi / T_{Q}^{\prime}$ for any $T_{Q}^{\prime} \leq T_{Q}$. Therefore, let $T_{Q}^{\prime}=T /(a M+1)$, where $M$ is the LCM of $\left\{M_{p}\right\}$, and $a$ is the smallest integer greater than zero such that $T_{Q}^{\prime} \leq T_{Q}$. Since the filters in Fig. 11 are all bandlimited to $\pi / T_{Q}$, they are also bandlimited to $\pi / T_{Q}^{\prime}$. In addition

$$
T_{p} / T_{Q}^{\prime}-1 / M_{p}=T /\left(M_{p} T_{Q}^{\prime}\right)-1 / M_{p}=a M / M_{p}
$$

which is an integer. Thus, if we regard $x_{c}(t)$ and the filters in Fig. 11 as bandlimited to $T_{Q}^{\prime}=T /(a M+1)$, then the condition of (30) holds. We can therefore apply the Interpolation Identity to each filterbank of the form of Fig. 11, resulting in the equivalent implementation of Fig. 12, where we substitute $T_{Q}^{\prime}$ for $T_{Q}$. In this case, $r[n]$ corresponds to uniform samples of $x_{c}(t)$ taken at the rate $\pi / T_{Q}^{\prime}$, which is higher than the Nyquist rate.

To summarize, for any sampling periods $T_{p}$, we can reconstruct a continuous-time signal from its $N$ th-order samples using $N$ discrete-time filters as in Fig. 12, where we substitute $T_{Q}^{\prime}=T /(a M+1)$ for $T_{Q}$, if necessary, with $a$ chosen so that $T_{Q}^{\gamma} \leq T_{Q}$.

\section{Generating New Classes of Sampling Strategies}

In the previous sections, we used the Interpolation Identity to obtain discrete-time filterbank structures for reconstruction of a continuous-time signal from generalized and nonuniformly spaced samples. In this section, we show how the filterbank interpretation suggests new sampling strategies.

The Interpolation Identity can be used to convert the reconstruction (synthesis) part of a continuous-time filterbank to an equivalent discrete-time filterbank followed by impulse modulation and lowpass filtering. Similarly, we can convert the sampling (analysis) part of a filterbank using the equivalence of

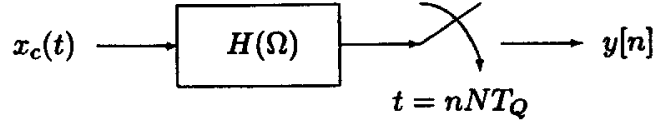

(a)

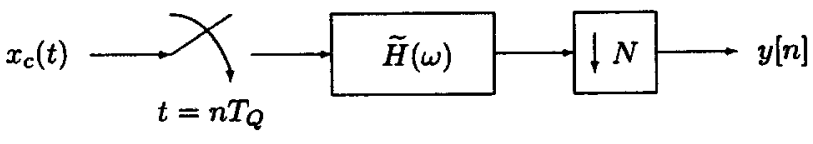

(b)

Fig. 13. Sampling equivalence.

Fig. 13(a) and (b) for any $x_{c}(t), h(t)$ bandlimited to $W=$ $\pi / T_{Q}$, where

$$
\tilde{H}(\omega)=H\left(\frac{\omega}{T_{Q}}\right), \quad|\omega| \leq \pi .
$$

The equivalence of Fig. 13 follows in a straightforward way by noting that sampling a continuous-time signal at one- $N$ th of the Nyquist rate can be realized by sampling the signal at the Nyquist rate followed by decimation by a factor of $N$. We can then apply the well-known results [7] regarding discrete-time processing of a continuous-time signal to replace the continuous-time filter by a discrete-time filter with frequency response given by (33), operating on Nyquist rate samples of the continuous-time signal.

The Interpolation Identity of Fig. 3 together with the equivalence of Fig. 13 enable us to convert any continuous-time filterbank to an equivalent discrete-time filterbank preceded by Nyquist rate sampling and followed by impulse modulation and lowpass filtering, and vice versa. Thus, any perfect reconstruction (PR) filterbank (i.e., a discrete-time analysis-synthesis filter bank for which the input and output are equal) can be converted to a continuous-time filterbank, which can then be interpreted in terms of sampling and reconstruction.

As an example, consider the PR filterbank of Fig. 14. The theory of PR filterbanks is well established (see, e.g., [12]), and closed-form solutions for the synthesis filters $H_{p}(\omega)$ given the analysis filters $F_{p}(\omega)$ are known. We can convert the analysis part of the filterbank to a sampling strategy by applying the equivalence of Fig. 13. This results in the sampling strategy depicted in Fig. 15, where the signal $x_{c}(t)$ is filtered by three continuous-time filters with frequency responses $F_{p}\left(\Omega T_{Q}\right), p=$ $0,1,2$, and the outputs are sampled at the corresponding rates. The reconstruction is obtained by applying the Interpolation 


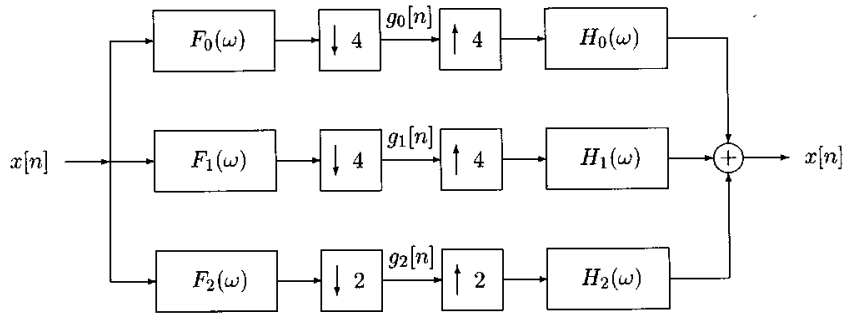

Fig. 14. Perfect reconstruction discrete-time filterbank.

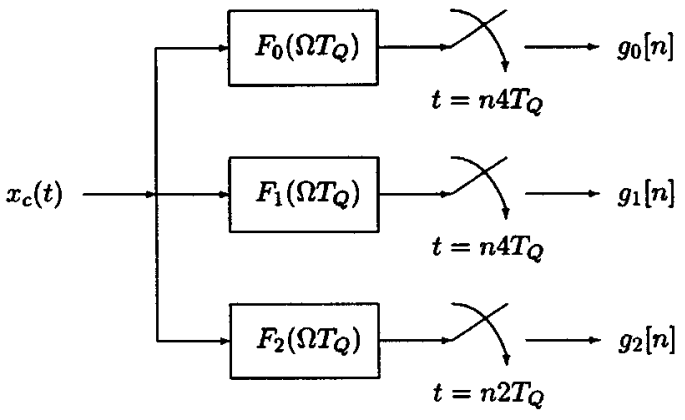

Fig. 15. Sampling procedure.

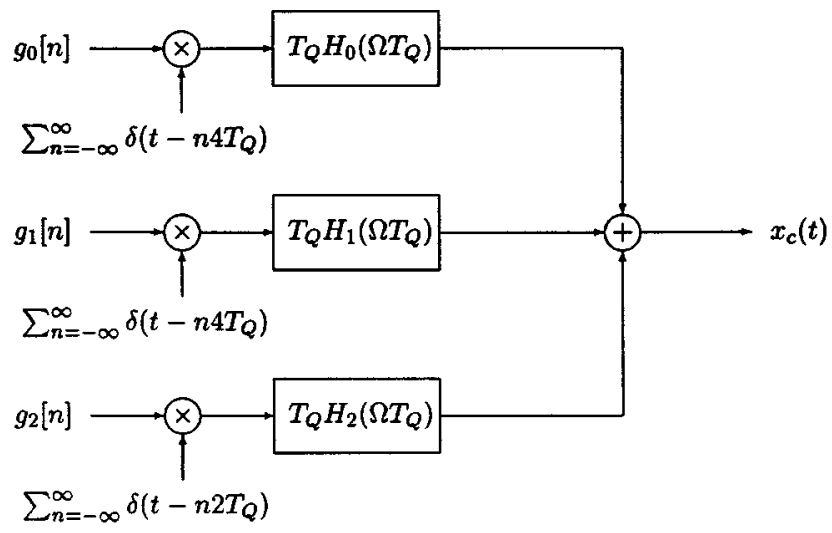

Fig. 16. Reconstruction using a continuous-time filterbank.

Identity of Fig. 3 to the synthesis part of the filterbank followed by impulse modulation and lowpass filtering, resulting in the reconstruction depicted in Fig. 16.

The sampling procedure of Fig. 15, together with the reconstruction of Fig. 16, constitute a generalization to Papoulis' well-known generalized sampling expansion (described in [8]). Papoulis showed that a bandlimited signal $x_{c}(t)$ is uniquely determined by the samples $g_{k}\left(n M T_{Q}\right)$ of the responses $g_{k}(t)$ of $M$ LTI filters with input $x_{c}(t)$ sampled at one- $M$ th of the Nyquist rate. By converting a PR filterbank with unequal decimation factors to a sampling and reconstruction scheme, we allow for different sampling rates of the filters outputs, thus generalizing Papoulis' theorem.

As an example, consider the sampling strategy discussed in Section III, namely, sampling a signal and its derivative at half the Nyquist rate. Such a sampling scheme may be employed in digital flight control, where the position, as well as the velocity of the aircraft, are recorded. In practical situations, we may be able to obtain readings of the velocity more frequently then readings of the position since the latter typically requires additional computation. In such cases, we can benefit from an alternative sampling strategy, where the position is recorded every $T_{p} T_{Q}$ seconds with $T_{p}>2$, and the velocity is recorded every $T_{p} /\left(T_{p}-1\right) T_{Q}$ seconds. Thus, we allow for a lower sampling rate of the position, which is compensated for by a higher sampling rate of the velocity.

Note that the fact that $x_{c}(t)$ is uniquely determined by samples of the outputs of $M$ filters sampled at different sampling rates can be shown using Papoulis' theorem by splitting the $p$ th branch, consisting of a continuous-time filter followed by uniform sampling with period $T_{p}$, into $T / T_{p}$ branches, where $T$ is the LCM of $\left\{T_{p}\right\}$. Each new branch will consist of filtering with a filter whose impulse response is the original impulse response shifted by a multiple of $T_{p}$ followed by uniform sampling with period $T$. The novelty in our generalization is in proving that the reconstruction is possible using a single filter for each branch instead of $T / T_{p}$ filters, as follows from Papoulis' theorem.

\section{CONCLUSION}

This paper introduces a filterbank interpretation of various sampling methods, thereby allowing for efficient implementation of the reconstruction from generalized samples as well as from a class of nonuniform samples. The main focus of this paper was on recurrent and $N$ th-order nonuniform sampling, for which a continuous-time filterbank implementation of the reconstruction was presented. Through the use of the Interpolation Identity derived in Section II, the continuous-time filterbanks were converted to equivalent discrete-time filterbanks followed by impulse modulation and lowpass filtering. The discrete-time filterbanks provide interpolation to uniform samples and can be implemented very efficiently exploiting the many known results regarding the implementation of filterbank structures. The block diagram equivalences formulated in this paper are general in the sense that they can be used to convert arbitrary continuous-time filterbanks to equivalent discrete-time filterbanks, and vise versa. Presenting the reconstruction from generalized samples in terms of continuous-time filters and applying the Interpolation Identity leads to efficient implementations that inherently interpolate the uniform Nyquist samples of the signal. Furthermore, the equivalences provide additional insight into the sampling and reconstruction process, thus leading to a whole new class of sampling strategies.

\section{APPENDIX A \\ PROOF OF THE INTERPOLATION IDENTITY}

Referring to Fig. 2(a) and denoting the input to the filter $H_{l}(\Omega)$ by $s_{l}(t)$, we have

$$
\begin{aligned}
s_{l}(t) & =\sum_{n=-\infty}^{\infty} x_{c}(n T) e^{-j(2 \pi l / M) n} \delta(t-n T) \\
& =\sum_{n=-\infty}^{\infty} x_{c}(t) e^{-j(2 \pi l / M)(t / T)} \delta(t-n T) .
\end{aligned}
$$


Consequently

$$
S_{l}(\Omega)=\frac{1}{T} \sum_{k=-\infty}^{\infty} X_{c}\left(\Omega+\frac{2 \pi l}{M T}-\frac{2 \pi k}{T}\right)
$$

and

$$
Y_{c}(\Omega)=\frac{1}{T} \sum_{l=0}^{M-1} H_{l}(\Omega) \sum_{k=-\infty}^{\infty} X_{c}\left(\Omega+\frac{2 \pi l}{M T}-\frac{2 \pi k}{T}\right)
$$

Referring to Fig. 2(b), we denote the Fourier transforms of $x[n], x_{e}[n], y_{e}[n]$, and $y_{d}[n]$ by $X(\omega), X_{e}(\omega), Y_{e}(\omega)$, and $Y_{d}(\omega)$, respectively. The sequence $x_{c}(n T)$ is obtained by uniformly sampling $x_{c}(t)$ with period $T$, and thus

$$
X(\omega)=\frac{1}{T} \sum_{k=-\infty}^{\infty} X_{c}\left(\frac{\omega}{T}-\frac{2 \pi k}{T}\right) .
$$

Using the frequency domain input-output relations of the expander and the decimator [7], we have

$$
X_{e}(\omega)=X\left(\frac{M T}{T_{Q}} \omega\right)=\frac{1}{T} \sum_{k=-\infty}^{\infty} X_{c}\left(\frac{M \omega}{T_{Q}}-\frac{2 \pi k}{T}\right)
$$

and

$$
\begin{aligned}
Y_{d}(\omega)= & \frac{1}{M} \sum_{l=0}^{M-1} Y_{e}\left(\frac{\omega}{M}+\frac{2 \pi l}{M}\right) \\
= & \frac{1}{M T} \sum_{l=0}^{M-1} \tilde{H}\left(\frac{\omega}{M}+\frac{2 \pi l}{M}\right) \\
& \sum_{k=-\infty}^{\infty} X_{c}\left(\frac{\omega}{T_{Q}}+\frac{2 \pi l}{T_{Q}}-\frac{2 \pi k}{T}\right) .
\end{aligned}
$$

Since $T / T_{Q}-1 / M$ is required to be an integer, we may substitute $k^{\prime}=k-l\left(T / T_{Q}-1 / M\right)$ in (39), resulting in

$$
\begin{aligned}
Y_{d}(\omega)= & \frac{1}{M T} \sum_{l=0}^{M-1} \tilde{H}\left(\frac{\omega}{M}+\frac{2 \pi l}{M}\right) \\
& \sum_{k^{\prime}=-\infty}^{\infty} X_{c}\left(\frac{\omega}{T_{Q}}+\frac{2 \pi l}{M T}-\frac{2 \pi k^{\prime}}{T}\right) .
\end{aligned}
$$

Next, we relate the transform of the output $Y_{c}(\Omega)$ to $Y_{d}(\omega)$. Since $y_{c}(t)$ is obtained by lowpass filtering a uniformly spaced impulse train with impulse train values $y_{d}[n]$ and period $T_{Q}[7]$

$$
Y_{c}(\Omega)= \begin{cases}T_{Q} Y_{d}\left(T_{Q} \Omega\right), & |\Omega| \leq W \\ 0, & \text { otherwise }\end{cases}
$$

Substituting (40) in (41) we have

$$
\begin{aligned}
Y_{c}(\Omega)= & \frac{T_{Q}}{M T} \sum_{l=0}^{M-1} \tilde{H}\left(\frac{T_{Q} \Omega}{M}+\frac{2 \pi l}{M}\right) \\
& \sum_{k=-\infty}^{\infty} X_{c}\left(\Omega+\frac{2 \pi l}{M T}-\frac{2 \pi k}{T}\right), \quad|\Omega| \leq W .
\end{aligned}
$$

Comparing (42) with (36), we conclude that the outputs of Fig. 2(a) and (b) are equal if

$$
H_{l}(\Omega)=\frac{T_{Q}}{M} \tilde{H}\left(\frac{T_{Q} \Omega}{M}+\frac{2 \pi l}{M}\right), \quad|\Omega| \leq W
$$

for $l=0,1, \cdots, M-1$. Equation (43) specifies how to choose $\tilde{H}(\omega)$ so that the outputs are equal. Specifically

$$
\begin{aligned}
\tilde{H}(\omega)=\frac{M}{T_{Q}} H_{l}\left(\frac{M \omega}{T_{Q}}-\frac{2 \pi l}{T_{Q}}\right) \\
\\
\frac{(2 l-1) \pi}{M} \leq \omega \leq \frac{(2 l+1) \pi}{M} .
\end{aligned}
$$

Since $H_{l}(\Omega)=0$ for $W \leq|\Omega|, H_{l}\left((M \omega-2 \pi l) / T_{Q}\right)=0$ outside $(2 l-1) \pi / M \leq \omega \leq(2 l+1) \pi / M$. We can therefore combine the $M$ equations represented by (44) for $l=$ $0,1, \cdots, M-1$ into the single equation

$$
\begin{array}{r}
\tilde{H}(\omega)=\frac{M}{T_{Q}} \sum_{l=0}^{M-1} H_{l}\left(\frac{M \omega}{T_{Q}}-\frac{2 \pi l}{T_{Q}}\right) \\
-\frac{\pi}{M} \leq \omega \leq 2 \pi-\frac{\pi}{M}
\end{array}
$$

or

$$
\begin{aligned}
\tilde{H}(\omega)= & \frac{M}{T_{Q}} \sum_{l=0}^{M-1}\left(H_{l}\left(\frac{M \omega}{T_{Q}}-\frac{2 \pi l}{T_{Q}}\right)\right. \\
& \left.+H_{l}\left(\frac{M \omega}{T_{Q}}-\frac{2 \pi(l-M)}{T_{Q}}\right)\right), \quad|\omega| \leq \pi .
\end{aligned}
$$

Choosing $\tilde{H}(\omega)$ according to (46) guarantees that the outputs of Fig. 2(a) and (b) are equal, establishing the identity.

APPENDIX B

PROOF OF THE RECONSTRUCTION FORMULA FROM RECURRENT NONUNIFORM SAMPLES (11)

Substituting (10) in (9) we have

$$
\begin{aligned}
G(t)=t & \prod_{\substack{n=-\infty \\
n \neq 0}}^{\infty}\left(1-\frac{t}{n T}\right) \prod_{n=-\infty}^{\infty}\left(1-\frac{t}{n T+t_{1}}\right) \cdots \\
& \prod_{n=-\infty}^{\infty}\left(1-\frac{t}{n T+t_{N-1}}\right) .
\end{aligned}
$$

Each one of the products in (47) converges to a constant times $\sin \left(\pi\left(t-t_{p}\right) / T\right)$. This can be shown as follows. The function $\sin (\pi t / T)$ can be expressed as an infinite product ([11, p. 114]):

$$
\sin (\pi t / T)=k t \prod_{\substack{n=-\infty \\ n \neq 0}}^{\infty}\left(1-\frac{t}{n T}\right)
$$

where $k$ is a constant. Thus

$$
\begin{aligned}
& \sin \left(\pi\left(t-t_{p}\right) / T\right) \\
& \quad=k\left(t-t_{p}\right) \prod_{\substack{n=-\infty \\
n \neq 0}}^{\infty}\left(1-\frac{t-t_{p}}{n T}\right)
\end{aligned}
$$




$$
\begin{aligned}
& =-k \prod_{n=-\infty}^{\infty}\left(n T-t+t_{p}\right) \prod_{\substack{n=-\infty \\
n \neq 0}}^{\infty} \frac{1}{n T} \\
& =-k t_{p} \prod_{n=-\infty}^{\infty}\left(1-\frac{t}{n T+t_{p}}\right) \prod_{\substack{n=-\infty \\
n \neq 0}}^{\infty} \frac{n T+t_{p}}{n T} \\
& =-k t_{p} \prod_{n=-\infty}^{\infty}\left(1+\frac{t_{p}}{n T}\right) \prod_{n=-\infty}^{\infty}\left(1-\frac{t}{n T+t_{p}}\right) \\
& =-\sin \left(\pi t_{p} / T\right) \prod_{n=-\infty}^{\infty}\left(1-\frac{t}{n T+t_{p}}\right) \\
& =\tilde{k} \prod_{n=-\infty}^{\infty}\left(1-\frac{t}{n T+t_{p}}\right) .
\end{aligned}
$$

Therefore, (47) can be rewritten as

$$
G(t)=c \prod_{p=0}^{N-1} \sin \left(\pi\left(t-t_{p}\right) / T\right)
$$

with $c$ a constant. Differentiating (50) and evaluating the derivative at $t=t_{p}+n T$, we have

$$
\begin{aligned}
G^{\prime}\left(t_{p}+n T\right) & =c \frac{\pi}{T} \cos (\pi n) \prod_{\substack{q=0 \\
q \neq p}}^{N-1} \sin \left(\pi\left(t_{p}+n T-t_{q}\right) / T\right) \\
& =c \frac{\pi}{T}(-1)^{n N} \prod_{\substack{q=0 \\
q \neq p}}^{N-1} \sin \left(\pi\left(t_{p}-t_{q}\right) / T\right) .
\end{aligned}
$$

Substituting (10), (50), and (51) in (8) results in the reconstruction formula given by (11).

Note, that Yen derives the same reconstruction formula in [16]. However, his derivation does not rely on Theorem 1 and is considerably more complex then the derivation presented here.

\section{APPENDIX C}

PROOF OF THE RECONSTRUCTION FORMULA FROM $N$ TH-ORDER NONUNIFORM SAMPLES (23)

For this case, (8) can be written as

$$
x_{c}(t)=\sum_{p=0}^{N-1} \sum_{n=-\infty}^{\infty} x_{c}\left(n T_{p}+t_{p}\right) \frac{G(t)}{G^{\prime}\left(n T_{p}+t_{p}\right)\left(t-n T_{p}-t_{p}\right)} .
$$

Substituting (22) in (9) and using (49), we conclude that

$$
G(t)=k \prod_{p=0}^{N-1} \sin \left(\pi\left(t-t_{p}\right) / T_{p}\right)
$$

with $k$ a constant. Differentiating (53) and evaluating the derivative at $t=n T_{p}+t_{p}$, we have

$$
\begin{aligned}
& G^{\prime}\left(t_{p}+n T\right) \\
& \quad=k \frac{\pi}{T_{p}} \cos (\pi n) \prod_{\substack{q=0 \\
q \neq p}}^{N-1} \sin \left(\pi\left(n T_{p}+t_{p}-t_{q}\right) / T_{q}\right)
\end{aligned}
$$

$$
=k \frac{\pi}{T_{p}}(-1)^{n} \prod_{\substack{q=0 \\ q \neq p}}^{N-1} \sin \left(\pi\left(t_{p}-t_{q}\right) / T_{q}+\pi n T_{p} / T_{q}\right) .
$$

The reconstruction formula given by (23) follows from substituting (53) and (54) in (52).

\section{APPENDIX D}

PROOF OF THE FILTER BANK REPRESENTATION OF $g_{p}(t)(25)$

From the definition of $f_{p k}(t)$ [see (27)] and the relation $\sin (t-k \pi)=(-1)^{k} \sin (t), g_{p}(t)$ [defined in (24)] can be written as

$$
g_{p}(t)=\sum_{k=-\infty}^{\infty} x_{c}\left(k T_{p}+t_{p}\right) f_{p k}\left(t-k T_{p}-t_{p}\right) .
$$

Note from (27) that $f_{p k}(t)=f_{p\left(k+M_{p}\right)}(t)$. Thus, we further express (55) as

$$
\begin{aligned}
g_{p}(t)= & \sum_{l=0}^{M_{p}-1} \sum_{k=-\infty}^{\infty} x_{c}\left(\left(k M_{p}+l\right) T_{p}+t_{p}\right) \\
& \cdot f_{p l}\left(t-\left(k M_{p}+l\right) T_{p}-t_{p}\right) \\
= & \sum_{l=0}^{M_{p}-1} \sum_{k=-\infty}^{\infty} \sum_{r=-\infty}^{\infty} \delta\left[k-l-r M_{p}\right] x_{c}\left(k T_{p}+t_{p}\right) \\
& \cdot f_{p l}\left(t-k T_{p}-t_{p}\right) .
\end{aligned}
$$

Using the equality

$$
\sum_{r=-\infty}^{\infty} \delta[k-l-r M]=\frac{1}{M} \sum_{m=0}^{M-1} e^{-j(2 \pi m / M)(k-l)}
$$

we rewrite $g_{p}(t)$ as

$$
\begin{aligned}
g_{p}(t)= & \frac{1}{M_{p}} \sum_{l=0}^{M_{p}-1} \sum_{k=-\infty}^{\infty} \sum_{m=0}^{M_{p}-1} x_{c}\left(k T_{p}+t_{p}\right) \\
& \cdot e^{-j\left(2 \pi m / M_{p}\right)(k-l)} f_{p l}\left(t-k T_{p}-t_{p}\right) \\
= & \sum_{m=0}^{M_{p}-1} \sum_{k=-\infty}^{\infty} x_{c}\left(k T_{p}+t_{p}\right) e^{-j\left(2 \pi m / M_{p}\right) k} \\
& \sum_{l=0}^{M_{p}-1} \frac{1}{M_{p}} e^{j\left(2 \pi m / M_{p}\right) l} f_{p l}\left(t-k T_{p}-t_{p}\right) .
\end{aligned}
$$

Equation (58) can be expressed as a sum of $M_{p}$ convolutions. Specifically, $g_{p}(t)=\sum_{m=0}^{M_{p}-1} s_{p m}(t) * h_{p m}(t)$, where $h_{p m}(t)$ and $s_{p m}(t)$ are defined in (26) and (28), respectively.

\section{ACKNOWLEDGMENT}

The authors wish to thank A. Russell for many fruitful discussions.

\section{REFERENCES}

[1] F. J. Beutler, "Error-free recovery of signals from irregularly spaced samples," SIAM Rev., vol. 8, pp. 328-335, July 1966.

[2] C. Cenker, H. G. Feichtinger, and M. Herrmann, "Iterative algorithms in irregular sampling a first comparison of methods," in Proc. ICCCP, Phoenix, AZ, Mar. 1991. 
[3] I. Djokovic and P. P. Vaidyanathan, "New sampling therems for MRA subspaces," in Proc. ICASSP, vol. 2, 1995, pp. 1085-1088.

[4] D. I. Jagerman and L. J. Fogel, "Some general aspects of the sampling theorem," IRE Trans. Inform. Theory, vol. IT-2, pp. 139-146, Dec. 1956.

[5] A. J. Jerri, "The Shannon sampling theorem-Its various extensions and applications: A tutorial review," Proc. IEEE, vol. 65, pp. 1565-1596, Nov. 1977.

[6] F. Marvasti, M. Analoui, and M. Gamshadzahi, "Recovery of signals from nonuniform samples using iterative methods," IEEE Trans. Signal Processing, vol. 39, pp. 872-877, Apr. 1991.

[7] A. V. Oppenheim, R. W. Schafer, and J. R. Buck, Discrete-Time Signal Processing, 2nd ed. Englewood Cliffs, NJ: Prentice-Hall, 1999.

[8] A. Papoulis, "Generalized sampling expansion," IEEE Trans. Circ. Syst., vol. CAS-24, pp. 652-654, Nov. 1977.

[9] E. I. Plotkin, M. N. S. Swamy, and Y. Yoganandam, "A novel iterative method for the reconstruction of signals from nonuniformly spaced samples," Signal Process., vol. 37, pp. 203-213, 1994.

[10] N. Sayiner, H. V. Sorensen, and T. R. Viswanathan, "A level-crossing sampling scheme for A/D conversion," IEEE Trans. Circ. Syst. II, vol. 43, pp. 335-339, Apr. 1996.

[11] E. C. Titchmarsh, The Theory of Functions, 2nd ed. Oxford, U.K.: Oxford Univ. Press, 1939

[12] P. P. Vaidyanathan, Multirate Systems and Filter Banks. Englewood Cliffs, NJ: Prentice-Hall, 1993.

[13] P. P. Vaidyanathan and S. M. Phoong, "Reconstruction of sequences from nonuniform samples," in Proc. ISCAS, vol. 1, 1995, pp. 601-604.

[14] R. G. Willey, "Recovery of bandlimited signals from unequally spaced samples," IEEE Trans. Commun., vol. COMM-26, pp. 135-137, Jan. 1978 .

[15] K. Yao and J. B. Thomas, "On some stability and interpolatory properties of nonuniform sampling expansions," IEEE Trans. Circuit Theory, vol CT-14, pp. 404-408, Dec. 1967.

[16] J. L. Yen, "On nonuniform sampling of bandwidth-limited signals," IEEE Trans. Circuit Theory, vol. CT-3, pp. 251-257, Dec. 1956.

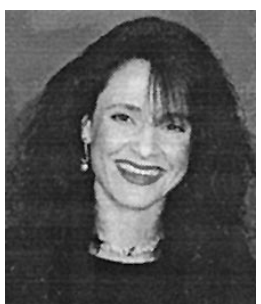

Yonina C. Eldar (S'98) received the B.Sc. degree in physics in 1995 and the B.Sc. degree in electrical engineering in 1996, both from Tel-Aviv University (TAU), Tel-Aviv, Israel. She is currently pursuing the $\mathrm{Ph} . \mathrm{D}$. degree in electrical engineering at the Massachusetts Institute of Technology (MIT), Cambridge.

From 1992 through 1996, she was in the program for outstanding students at TAU. Since 1998, she has been with the Digital Signal Processing Group, MIT She has served as a Teaching Assistant for classes in linear systems, digital signal processing, and statistical signal processing. Her current research interests are in the general area of signal processing.

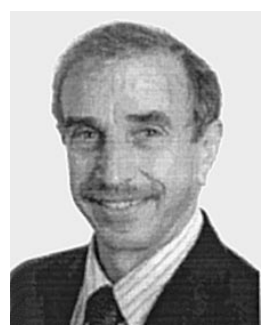

Alan V. Oppenheim (F'77) received the S.B. and S.M degrees in 1961 and the Sc.D. degree in 1964, all in electrical engineering, from the Massachusetts Institute of Technology (MIT), Cambridge. He was also the recipient of an honorary doctorate degree from Tel-Aviv University, Tel-Aviv, Israel, in 1995.

In 1964, he joined the faculty at MIT, where he is currently the Ford Professor of Engineering and a MacVicar Faculty Fellow with the Department of Electrical Engineering and Computer Science. Since 1967, he has also been affiliated with MIT Lincoln Laboratory, Lexington, and, since 1977, with the Woods Hole Oceanographic Institution, Woods Hole, MA. His research interests are in the general area of signal processing and its applications. He is coauthor of the widely used textbooks Discrete-Time Signal Processing and Signals and Systems. He is also Editor of several advanced books on signal processing.

Dr. Oppenheim is a member of the National Academy of Engineering and a member of Sigma Xi and Eta Kappa Nu. He has been a Guggenheim Fellow and a Sackler Fellow at Tel-Aviv University. He has also received a number of awards for outstanding research and teaching including the IEEE Education Medal, the IEEE Centennial Award, the Society Award, the Technical Achievement Award, and the Senior Award of the IEEE Society on Acoustics, Speech, and Signal Processing. He has also received a number of awards at MIT for excellence in teaching, including the Bose Award and the Everett Moore Baker Award. 